\title{
Rigid Body Motions and Local Compliance Response during Impact of Two Deformable Spheres
}

\author{
Akuro Big-Alabo ${ }^{1}$ \\ ${ }^{1}$ Department of Mechanical Engineering, Faculty of Engineering, University of Port Harcourt, Choba, Nigeria \\ Correspondence: Akuro Big-Alabo, Department of Mechanical Engineering, Faculty of Engineering, University of \\ Port Harcourt, Choba, Rivers State, Nigeria. Tel: 234-816-468-3549. E-mail: akuro.big-alabo@uniport.edu.ng
}

Received: November 13, 2017

Accepted: November 29, 2017 Online Published: December 20, 2017

doi:10.5539/mer.v8n1p1

URL: https://doi.org/10.5539/mer.v8n1p1

\begin{abstract}
The impact of two hard deformable spheres is revisited with the aim of investigating the constituent rigid body motions and indentation response of each sphere during collision. The latter are determined theoretically and the theoretical solutions are validated by comparing with numerical solutions of the coupled nonlinear dynamic models for impact of two hard deformable spheres. For elastic impact events, normalized tabulated solutions are derived using the Force Indentation Linearisation Method (FILM) and the tabulated solutions can be used to generate actual rigid body motions and indentation histories for each of the colliding spheres without need for numerical or finite element solutions. The analysis shows that the rigid body motion and local compliance response of each sphere depend on: (a) ratio of mass of sphere to effective mass of impact system, and (b) ratio of initial velocity of sphere to initial relative velocity of impact system. Finally, the 2-D collision problem is discussed and a simple procedure to determine the unique solution of all four unknowns is presented.
\end{abstract}

Keywords: elastic impact, elastoplastic impact, spherical impact, FILM, 2-D collision

\section{Introduction}

Collision of rigid bodies is a phenomenon of immense engineering importance. Rigid body collisions occur in physical processes such as granular flow (Campbell, 2006), multi-body dynamics (Machado et al., 2012) and projectile impact dynamics (Goldsmith, 2001). A classical case, with general implications, used to study rigid body collisions is the impact of two deformable spheres. When two deformable spheres come into non-conformal contact at a relative velocity, a local deformation occurs in the region of contact. The kinetic energy of the relative velocity is converted to a compressive pressure that deforms the spheres during the impact loading stage. This continues until the relative velocity is zero and the total deformation is maximum; thereafter, the deformations on both spheres are reversed in the unloading stage. For elastic contact the reversal is complete, but for elastoplastic contact a permanent deformation is left on either or both spheres.

Experimental investigations on the impact of spheres have been conducted. Andrews (1930a) conducted pendulum experiments for the impact of two identical spheres in order to measure the contact duration, coefficient of restitution and permanent indentation during elastoplastic impact. Goldsmith (2001) summarized measurements of coefficient of restitution and contact duration for elastoplastic impact experiments conducted before 1960. Results for experiments on impact of identical spheres as well as spheres of different sizes and material composition were presented and discussed. More recent experiments on the impact of two identical spheres have been reported by Minamoto and Kawamura (2009; 2011). Experimental results for coefficient of restitution and contact duration were reported for low-velocity impacts (Minamoto \& Kawamura, 2009) and moderate-velocity impacts (Minamoto \& Kawamura, 2011), and the measurements were validated using FE simulation results. Also, experiments on impact of spheres on flat targets have been conducted (Kharaz \& Gorham, 2000) and are a special case of impact of two spheres i.e. the flat target may be considered as a sphere of infinite radius. Experimental investigations are usually limited in the information that can be obtained, but are useful for validating theoretical and FE models, from which more details of the impact process can be realized.

Details of an impact event that are difficult to measure experimentally, such as indentation history, can be readily obtained by solving the theoretical models governing the impact event. Also, the theoretical approach is a cost effective means of analyzing impact problems. The theoretical models for the impact of two deformable spheres are normally formulated from the inertial equation accounting for the dynamics of the spheres and the contact model 
accounting for the local compliance of the spheres. The theoretical model for normal elastic impact of two deformable spheres has been discussed by Johnson (1985) and the solution of the relative response in terms of the total indentation and contact force histories were plotted in a normalized form. Andrews (1930b) proposed theoretical models for normal elastoplastic impact of two identical spheres and used the models to predict contact duration and permanent indentation. The theoretical predictions obtained by Andrews (1930b) agreed with experiments to which they were compared. Du and Wang (2009) presented theoretical models for elastoplastic impact of two deformable spheres and used the models to predict the final velocities of the spheres after impact. Du and Wang (2009) validated their models using predictions from FE simulations. The main limitation of the above theoretical studies is that they have been conducted only to determine the total compliance response without considering the constituent compliance response or rigid body motions.

During the impact process, each colliding sphere undergoes a local compliance and at the same time experiences a rigid body motion. Hence, we define the constituent response of each sphere as the compliance history (i.e. indentation/indentation rate) and rigid body motion (i.e. rigid body displacement/velocity) of the sphere that contributes to the total response of both spheres. The total response of both spheres consists of the relative rigid body motion which is identical to the total compliance response i.e. sum of the constituent compliance responses. To get a complete picture of the impact process of two hard deformable spheres, the constituent responses of each sphere must be known and is necessary for understanding of important physical processes. For example, it may be necessary to determine whether either or both colliding bodies undergo elastoplastic indentation during collision, and to know the extent of permanent indentation realized on each colliding body, if any. Hence, such information can assist to design experiments in which it is desired that one of the colliding bodies does not undergo elastoplastic indentation (Kharaz \& Gorham, 2000). Another situation where knowledge of the response of individual objects in collision is important is for accurate motion tracking of freely colliding particles as found in atomic collisions and granular flow processes. Finally, knowing the constituent responses of each sphere aids better understanding of the utilization mechanism of the total collision energy.

Studies to determine the constituent response histories during impact of two deformable spheres have not been conducted (Coaplen et al., 2004). Theoretical models governing the impact dynamics of two spheres have been used only to determine the total compliance response (i.e. histories of total indentation and relative velocity) and other impact parameters that are directly obtained from the total compliance response (i.e. contact duration and impact force history). Theoretical or FE solutions for the constituent response histories of individual spheres during impact does not to appear in the literature except for the special case of two identical spheres (Andrews, 1930b). Even for the case of identical spheres, the constituent rigid body motions of the spheres is yet to be investigated.

It is the intention of this paper to address this identified gap in the understanding of rigid body impact by presenting theoretical solutions for computing the constituent responses during binary impact of spheres. The main aim is not to present new impact models but to use existing models to determine and analyze the constituent response of each colliding sphere that determines the total response of both spheres. To achieve this, a recently developed analytical method is uniquely applied to provide solutions to the constituent compliance and rigid body response of each sphere. The analytical solution approach is demonstrated by considering elastic and elastoplastic impact case studies. Additionally, tabulated normalized solutions are derived to determine constituent responses during normal collision of two elastic spheres. The tabulated solutions can be easily used to generate actual impact profiles without need for FE solutions or numerical integration. Finally, solution to the 2-D collision problem is presented and discussed based on analysis of the constituent rigid body motions.

\section{Dynamics of Normal Impact of Two Deformable Spheres}

The present analysis is limited to the impact of two hard deformable spheres with smooth surface and experiencing a non-conformal contact during impact. More details on non-conformal contact can be found in Johnson (1985). The scope of the present study does not cover collision of two soft spheres or hard-soft spheres.

Figures 1(a-d) depict the elastic collision of two hard deformable spheres with smooth surfaces. Figures 1(a) and 1(c) show what happens before and after collision, while Figure 1(b) depicts the collision process. The directions of the final velocities are dependent on the initial velocities and masses of the colliding spheres and therefore, the directions shown in Figure 1(c) are just for the purpose of illustration and may not represent the actual final directions. Whereas the states of the spheres in Figures 1(a) and 1(c) are independent of time, the state in Figure 1(b) is time dependent. The section marked by the rectangle in Figure 1(b) is amplified in Figure 1(d) and shows the constituent indentations of the colliding spheres, $\delta_{1}$ and $\delta_{2}$, the total indentation, $\delta$, and the impact force, $F(\delta)$, which is estimated using a contact model. 
The impact of two deformable spheres gives rise to rigid body motions as well as local compliance in the contact region (see Figure 2). The rigid body motions consist of the displacement and velocity of the center of mass of each sphere and those of the contact plane. The local compliance consists of the indentation and indentation rate on each sphere or the total of these. Whereas the local compliance are scalar quantities, the rigid body motions are vector quantities. The implication is that a sign convention is necessary for the rigid body motions. Two rigid body motions are distinguished, namely: constituent and relative motions. The former represents the motion of a sphere considered independent of the other colliding sphere, while the latter represents the motions of both spheres considered together. In the following analysis, two sign conventions are adopted for the direction of the rigid body motions depending on whether the frame of reference is the constituent motion of a sphere or the relative motion of both spheres. For the constituent motion, the direction is positive when the motion is towards the contact plane and negative when the motion is away from the contact plane. On the other hand, the direction of a motion in the relative case is positive when it is in the positive $\mathrm{x}$-direction. The opposite direction is negative.

(a)

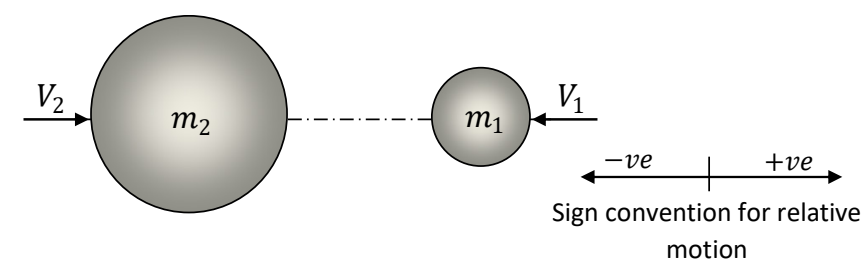

(b)

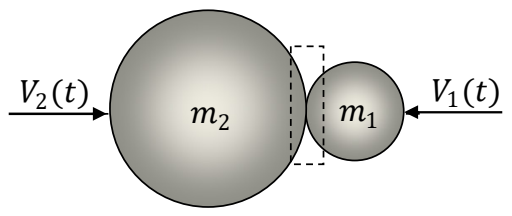

(c)
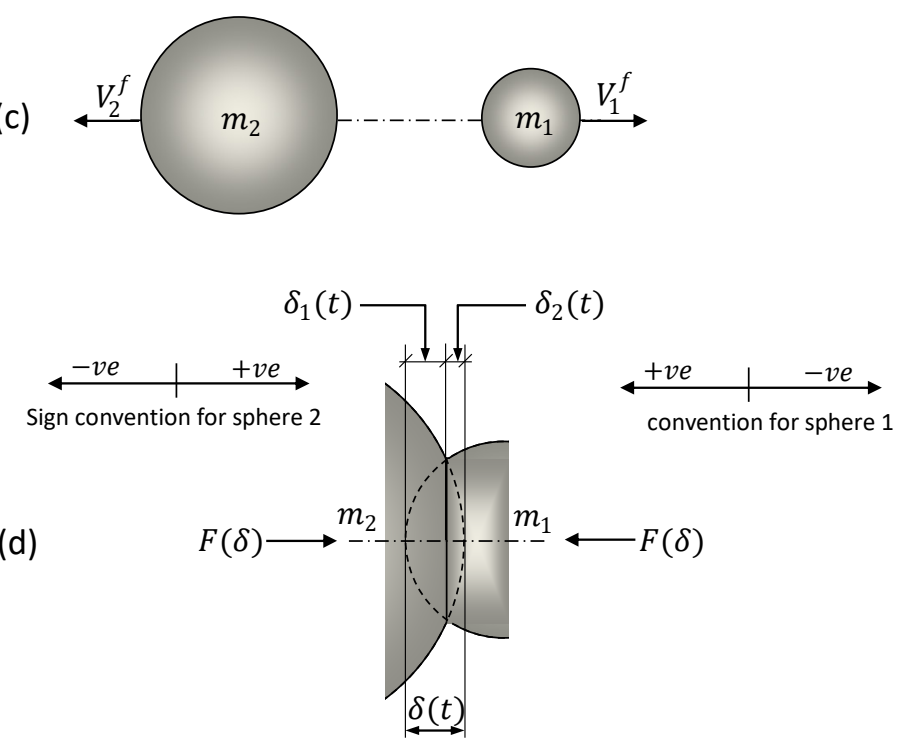

Figure 1. Elastic collision of two hard deformable spheres (a) before impact (b) during impact (c) after impact (d) amplification of contact zone

During normal impact of two hard deformable spheres the total indentation, $\delta$, is equal to the sum of the constituent indentations of both spheres i.e. $\delta=\delta_{1}+\delta_{2}$ (see Figures 1(d) and 2). The total indentation is also equal to the relative motion between the spheres and is expressed in terms of the displacements of the centers of mass of the spheres i.e. $\delta=d_{1}^{c}+d_{2}^{c}$ (see Figure 2), where the superscript indicates center of mass. This does not mean that $\delta_{1}=$ $d_{1}^{c}$ and $\delta_{2}=d_{2}^{c}$. The motion of the center of mass of each sphere is resisted by the impact force so that the acceleration of the center of mass is equal and opposite to the impact force. Hence,

$$
m_{1} \ddot{d}_{1}^{c}=-F(\delta)
$$


Equations $(1 \mathrm{a}, \mathrm{b})$ can be combined as:

$$
m_{2} \ddot{d}_{2}^{c}=-F(\delta)
$$

$$
m \ddot{\delta}=-F(\delta)
$$

where $m=\left(1 / m_{1}+1 / m_{2}\right)^{-1}$ is the effective mass of the impact system. The initial conditions to equations (1) and (2) are: $d_{1}^{c}(0)=d_{2}^{c}(0)=0, \delta(0)=0, \dot{d}_{1}^{c}(0)=V_{1}, \dot{d}_{2}^{c}(0)=V_{2}$ and $\dot{\delta}(0)=V_{0, \text { rel }}$, where $V_{0, \text { rel }}$ is the initial relative velocity. Equations $(1 \mathrm{a}, \mathrm{b})$ are generally coupled nonlinear ODEs with $\delta=d_{1}^{c}+d_{2}^{c}$ as the coupling parameter.

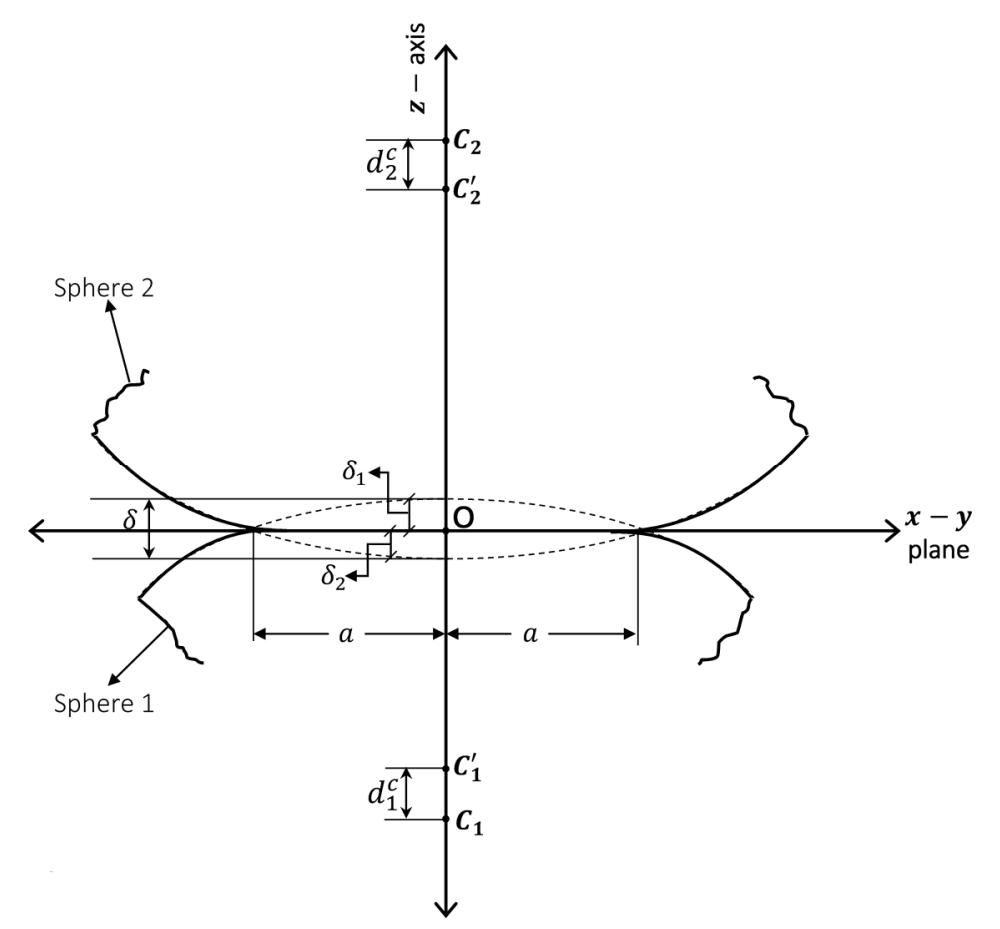

Figure 2. Quasi-static contact during impact of two hard deformable spheres

The local deformation of both spheres occurs at the plane of contact, which is the plane joining the surfaces of both spheres. The contact plane can be considered as the center of inertia of the impact system (Stronge, 2000) and moves at a constant velocity in the direction of the sphere with greater momentum. This motion is independent of the local compliance of the spheres (Stronge, 2000) and is determined from the momentum balance before and during collision as shown.

$$
V_{c p}=\frac{m_{1} V_{1}+m_{2} V_{2}}{m_{1}+m_{2}}
$$

where $V_{c p}$ is the contact plane velocity. Hence, the indentation and indentation rate of each sphere is determined as the relative motion between its center of mass and the contact plane i.e.

$$
\begin{gathered}
\delta_{i}(t)=d_{i}^{c}(t) \pm V_{c p} t \\
\dot{\delta}_{i}(t)=\dot{d}_{i}^{c}(t) \pm V_{c p}
\end{gathered}
$$

where $i=1,2$ for spheres 1 and 2 . In equations (4a,b), the sign is negative if the contact plane and sphere $i$ are initially moving in the same direction otherwise it is positive.

\section{Solution of Dynamic Models for Normal Impact of Two Deformable Spheres}

Equations (1a,b) were solved analytically using the force-indentation linearisation method (FILM) and also numerically in order to validate the analytical solution. The FILM is much easier to implement compared to FE or numerical methods, and as is shown later in this section, is capable of uncoupling the dynamic equations for the rigid body motions of the spheres (i.e. equation $(1 \mathrm{a}, \mathrm{b})$ ); thereby making it easy to obtain solutions for the constituent responses of the spheres. The concept and formulation of the FILM are explained in Big-Alabo et al. $(2015 ; 2017)$ and 
are not repeated here for brevity. According to the FILM, the linearized impact force for each $n$ discretization of a nonlinear compliance model can be expressed as (Big-Alabo et al., 2017):

$$
F_{r s}=K_{r s}\left(\delta_{r s}-\delta_{r}\right)+F\left(\delta_{r}\right)
$$

where $K_{r s}=\left[F\left(\delta_{s}\right)-F\left(\delta_{r}\right)\right] /\left(\delta_{s}-\delta_{r}\right) ; r$ and $s$ are respectively the initial and end states of each discretization. Substituting equation (5) in (2),

The solution to equation (6) is given as

$$
m \ddot{\delta}_{r s}+K_{r s} \delta_{r s}=K_{r s} \delta_{r}-F\left(\delta_{r}\right)
$$

$$
\begin{gathered}
\delta_{r s}(t)=R_{r s} \sin \left(\omega_{r s} t+\varphi_{r s}\right)+C_{r s} \\
\dot{\delta}_{r s}(t)=\omega_{r s} R_{r s} \cos \left(\omega_{r s} t+\varphi_{r s}\right)
\end{gathered}
$$

where $\omega_{r s}=\sqrt{K_{r s} / m}, \quad R_{r s}=\left[\left(\delta_{r}-C_{r s}\right)^{2}+\left(\dot{\delta}_{r} / \omega_{r s}\right)^{2}\right]^{1 / 2}, \quad \varphi_{r s}=\tan ^{-1}\left[\omega_{r s}\left(\delta_{r}-C_{r s}\right) / \dot{\delta}_{r}\right]-\omega_{r s} t_{r} \quad$ and $C_{r s}=\left[K_{r s} \delta_{r}-F\left(\delta_{r}\right)\right] / K_{r s}$. The initial conditions for each discretization are $\delta_{r}=\delta\left(t_{r}\right)=\delta_{\text {ini }}+r\left(\delta_{n}-\delta_{\text {ini }}\right) / n$ and $\dot{\delta}_{r}=\dot{\delta}\left(t_{r}\right)= \pm\left[V_{\text {ini }}^{2}-(2 / m) \int_{\delta_{\text {ini }}}^{\delta_{r}} F(\delta) d \delta\right]^{1 / 2}$ where $\delta_{i n i} \leq \delta \leq \delta_{n}$ is the indentation range for the nonlinear impact stage considered, $V_{i n i}$ is the relative velocity at the onset of this impact stage and

$$
t_{r}=\left(\frac{\pi}{2} \pm \operatorname{ArcCos}\left[\left(\delta_{r}-C_{r s}\right) / R_{r s}\right]-\varphi_{r s}\right) / \omega_{r s}
$$

Equations $(3-8)$ have been derived previously (Big-Alabo et al., 2015; 2017) and are used to determine the total compliance response of both spheres. Here the FILM is extended to determine the constituent responses of each sphere as follows. In equation (5), the linearized force is expressed in terms of the total indentation, but the solution of the total indentation as a function of time has been derived in equation (7a). Hence, substituting equation (7a) in (5) gives the linearized impact force for each discretization as an explicit function of time:

$$
F_{r s}=K_{r s} R_{r s} \sin \left(\omega_{r s} t+\varphi_{r s}\right)
$$

Using equation (9) in (1a,b), the uncoupled equations describing the rigid body motion of each sphere are:

$$
\left(\ddot{d}_{i}^{c}\right)_{r s}=\left(K_{r s} R_{r s} / m_{i}\right) \sin \left(\omega_{r s} t+\varphi_{r s}\right) \quad \text { for } i=1,2
$$

Direct integration of equation (10) gives the rigid body velocity and displacement as:

and

$$
\left(\dot{d}_{i}^{c}\right)_{r s}=\left(\dot{d}_{i}^{c}\right)_{r}+\left(K_{r s} R_{r s} / m_{i} \omega_{r s}\right) \cos \left(\omega_{r s} t+\varphi_{r s}\right)-Y_{r s}
$$

$$
\left(d_{i}^{c}\right)_{r s}=\left(d_{i}^{c}\right)_{r}+\left(K_{r s} R_{r s} / m_{i} \omega_{r s}^{2}\right) \sin \left(\omega_{r s} t+\varphi_{r s}\right)+\left[\left(\dot{d}_{i}^{c}\right)_{r}-Y_{r s}\right] t-Z_{r s}
$$

respectively, where $\left(\dot{d}_{i}^{c}\right)_{r}=\dot{d}_{i}^{c}\left(t_{r}\right) ;\left(d_{i}^{c}\right)_{r}=d_{i}^{c}\left(t_{r}\right) ; Y_{r s}=\left(K_{r s} R_{r s} / m_{i} \omega_{r s}\right) \cos \left(\omega_{r s} t_{r}+\varphi_{r s}\right)$ and $Z_{r s}=$ $\left(K_{r s} R_{r s} / m_{i} \omega_{r s}^{2}\right) \sin \left(\omega_{r s} t_{r}+\varphi_{r s}\right)+\left[\left(\dot{d}_{i}^{c}\right)_{r}-Y_{r s}\right] t_{r}$. Applying equations $(7 \mathrm{a}, \mathrm{b})$ in equations $(11 \mathrm{a}, \mathrm{b})$ and noting that $\omega_{r s}^{2}=K_{r s} / m$, the solution of the rigid body velocity and displacement of each sphere can be written more elegantly as shown.

$$
\begin{gathered}
\left(\dot{d}_{i}^{c}\right)_{r s}=\left(\dot{d}_{i}^{c}\right)_{r}+\left(m / m_{i}\right)\left(\dot{\delta}_{r s}-\dot{\delta}_{r}\right) \\
\left(d_{i}^{c}\right)_{r s}=\left(d_{i}^{c}\right)_{r}+\left(m / m_{i}\right)\left(\delta_{r s}-\delta_{r}\right)+\left[\left(\dot{d}_{i}^{c}\right)_{r}-\left(m / m_{i}\right) \dot{\delta}_{r}\right]\left(t-t_{r}\right)
\end{gathered}
$$

The uncoupling of the rigid body response of each sphere was made possible because the FILM produces closed-form solutions that are explicit functions of time. An advantage of this is that the parameters that influence the constituent rigid body motions and compliance response of each sphere can be ascertained analytically. With the conventional numerical schemes, the response of each sphere cannot be uncoupled, the solution is more complicated and the parameters that influence the response of each sphere are difficult to determine. 


\section{Discussions of Results}

\subsection{Convergence of the FILM}

Previous studies (Big-Alabo et al., 2015; 2017) have discussed the inherent stability and convergence ability of the FILM but the convergence rate has not been tested. The purpose of this section is to perform a convergence test of the FILM algorithm in order to establish its convergence rate. The convergence rate of the FILM algorithm is tested here using an elastic impact event, for which the impact force is estimated using the Hertz contact model i.e. $F=K_{h} \delta^{3 / 2}$ where $K_{h}=(4 / 3) E R^{1 / 2}$. The FILM constants for elastic impact of two spheres are derived in concise from as:

$$
\left.\begin{array}{c}
K_{r s}=\gamma_{r s} K_{h}\left(\delta_{m} / n\right)^{\frac{1}{2}} \\
\omega_{r s}=\left(\frac{1.25 \gamma_{r s}}{\sqrt{n}}\right)^{\frac{1}{2}}\left(\frac{V_{0, r e l}}{\delta_{m}}\right) \\
C_{r s}=\frac{r}{n}\left(1-\frac{\sqrt{r}}{\gamma_{r s}}\right) \delta_{m} \\
R_{r s}=\left[\frac{r^{3}}{\left(n \gamma_{r s}\right)^{2}}+\frac{\sqrt{n}}{1.25 \gamma_{r s}}\left(1-\left(\frac{r}{n}\right)^{\frac{5}{2}}\right)\right]^{\frac{1}{2}} \delta_{m} \\
\dot{\delta}_{r}= \pm\left[1-\left(\frac{r}{n}\right)^{\frac{5}{2}}\right]^{\frac{1}{2}} V_{0, r e l} \\
\delta_{r}=\frac{r \delta_{m}}{n}
\end{array}\right\}
$$

where $\gamma_{r s}=s^{3 / 2}-r^{3 / 2} ; \delta_{m}=\left(5 m V_{0, r e l}^{2} / 4 K_{h}\right)^{2 / 5}$.

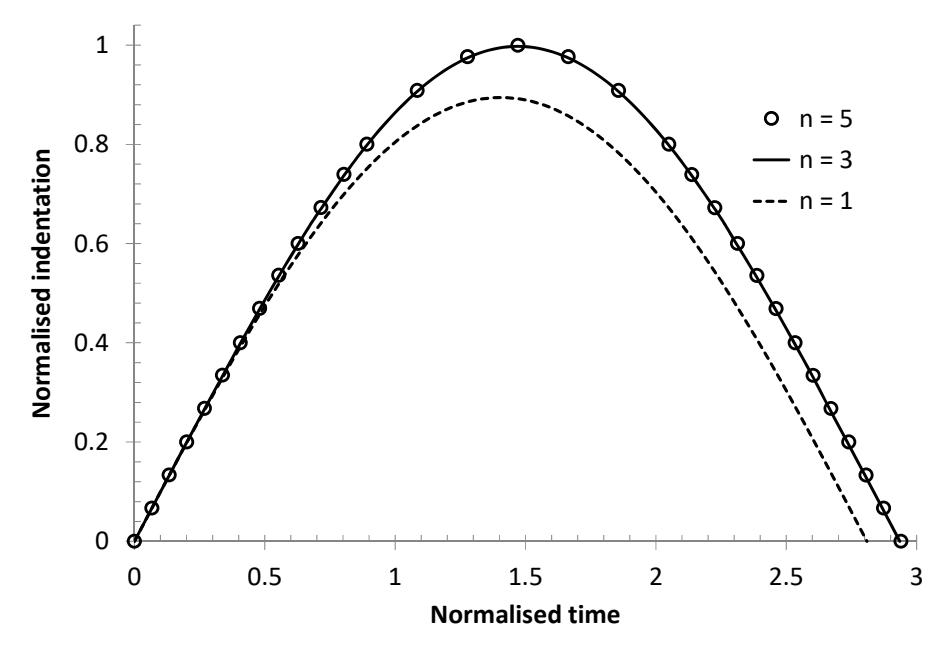

Figure 3. Convergence test for FILM solution using $\mathrm{n}=1,3,5$

A quick test of the convergence rate of the FILM can be conducted by considering the impact duration for elastic impact of two spheres. Goldsmith (2001) gave an accurate estimate of the impact duration as $t=2.9432\left(\delta_{m} / V_{0, r e l}\right)$. The FILM solution gives $t=2.9354\left(\delta_{m} / V_{0, \text { rel }}\right)$ for $n=3$ with error of $0.265 \%$ and $t=2.9405\left(\delta_{m} / V_{0, \text { rel }}\right)$ for $n=5$ with error of $0.092 \%$. This shows that a sufficiently accurate solution can be obtained with five discretization.

Detailed convergence test was conducted by comparing the indentation response for $n=1, n=3$ and $n=5$. This test is illustrated in Figure 3, which shows that the solution converges for $n=5$. Further comparison for $n=5$ and $n=25$, not shown here, reveals that there is no discernable improvement in the FILM solution for $n>5$. Note that the impact duration for $n=25$ is exactly the same as Goldsmith (2001).

\subsection{Normalized Response for Normal Elastic Impact of Two Deformable Spheres}

The dynamic models for normal elastic impact of two spheres are standard models and therefore, the response solutions can be normalized. The benefit in doing this is that the normalized solution can be easily used to generate actual impact profiles without need for numerical integration or FE solutions. Although normalized solutions have been presented for the total compliance responses during elastic collision of two spheres (e.g. Johnson, 1985) none has 
been presented for the constituent rigid body motions. The development of the tabulated normalized solutions for the constituent rigid body motions during elastic collision of two spheres is one of the main contributions of the present study. Tables A1 and A2 (see appendix) are tabulated normalized solutions for the total compliance response and the rigid body motions. These tabulated solutions were generated using $n=25$ and by extracting an additional solution at the mid-point of the time interval of the $25^{\text {th }}$ discretization.

The normalized total compliance responses are determined as: $\dot{\delta}_{r} / V_{0, r e l}= \pm\left[1-(r / n)^{5 / 2}\right]^{1 / 2} ; \delta_{r} / \delta_{m}=r / n$ and $F_{r} / F_{m}=(r / n)^{3 / 2}$, whereas the normalized rigid body motions are determined as:

$$
\begin{gathered}
\frac{\left(\dot{d}_{i}^{c}\right)_{r s}}{V_{0, r e l}}=\frac{\left(\dot{d}_{i}^{c}\right)_{r}}{V_{0, r e l}}+\left(\frac{m}{m_{i}}\right)\left(\frac{\dot{\delta}_{r s}}{V_{0, \text { rel }}} \mp \sqrt{1-\left(\frac{r}{n}\right)^{5 / 2}}\right) \\
\frac{\left(d_{i}^{c}\right)_{r s}}{\delta_{m}}=\frac{\left(d_{i}^{c}\right)_{r}}{\delta_{m}}+\left(\frac{m}{m_{i}}\right)\left(\frac{\delta_{r s}}{\delta_{m}}-\frac{r}{n}\right)+\left[\frac{\left(\dot{d}_{i}^{c}\right)_{r}}{V_{0, \text { rel }}}-\left(\frac{m}{m_{i}}\right) \dot{\delta}_{r}\right]\left(\bar{t}-\bar{t}_{r}\right)
\end{gathered}
$$

where $\bar{t}=\left(V_{0, r e l} / \delta_{m}\right) t$ and $\bar{t}_{r}=\left(V_{0, \text { rel }} / \delta_{m}\right) t_{r}$ are calculated using equations (8) and (13).

Columns 4 and 5 of Tables A1 and A2 show that the constituent rigid body motions, and by implication the constituent compliance responses (see equations $(4 a, b)$ ), depend on two ratios namely: (a) ratio of mass of sphere to effective mass of impact system, and (b) ratio of initial velocity of the sphere to initial relative velocity. Furthermore, the last row of Table A1 shows that the maximum indentation on each sphere can be calculated as:

$$
\left(\delta_{i}\right)_{m}=\left[1.47163\left(\frac{V_{i}}{V_{0, \text { rel }}}\right)-0.47163\left(\frac{m}{m_{i}}\right) \pm 1.47163\left(\frac{V_{c p}}{V_{0, \text { rel }}}\right)\right] \delta_{m}
$$

When the indentations on both spheres is maximum the rigid body velocities are equal to the contact plane velocity, so that the indentation rates of the spheres are momentarily equal to zero. This condition implies that $V_{i}-$ $\left(m / m_{i}\right) V_{0, r e l}=\mp V_{c p}$. The validity and applicability of the normalized tables is demonstrated with an elastic impact case study discussed next.

\subsection{Example on Normal Elastic Impact of Two Deformable Spheres}

Consider the normal elastic impact between two spheres of diameters $20[\mathrm{~mm}]$ and $40[\mathrm{~mm}]$ approaching each other from opposite directions (see Figure 1(a)) with initial velocities of $5.0[\mathrm{~mm} / \mathrm{s}]$ and $7.5[\mathrm{~mm} / \mathrm{s}]$ respectively. Each sphere is made of steel material with properties: $E=210[\mathrm{GPa}] ; \rho=7800\left[\mathrm{~kg} / \mathrm{m}^{3}\right] ; \sigma_{y}=1000[\mathrm{MPa}]$ and $v=0.3[-]$.

The initial velocities of the spheres were chosen so that the initial relative velocity (or impact velocity) is within the limit of elastic collision. Stronge's formulation for calculating the impact velocity that would cause yield (Stronge, 2000) was used to ensure the initial velocities of the spheres would result in elastic collision only.

Figures 4 and 5 show the rigid body motions obtained using the tabulated solutions and numerical integration carried out in Mathematica ${ }^{\mathrm{TM}}$. The tabulated solutions were implemented using a customized MS Excel spreadsheet. On the other hand, the numerical integration results were obtained by solving equation (2) first and using the solution of equation (2) to get the impact force solution. The latter was then substituted into equations $(1 \mathrm{a}, \mathrm{b})$ before integrating numerically to get the solution of the rigid body motions. Both solution methods are shown produce matching results thus validating the tabulated solutions. These results predict that each sphere has undergone a rigid body displacement of $1.047[\mu \mathrm{m}]$ at the end of impact process. Though the rigid body displacements at the end of the impact process for this example seems negligible, the present observation that binary collision results in rigid body displacements is contrary to the usual assumption that the colliding spheres are momentarily stationary during impact (Chatterjee, 1997). For instance, if spheres 1 and 2 are considered to move initially in the negative $x$-direction with velocities of $100.0125[\mathrm{~m} / \mathrm{s}]$ and $100.0[\mathrm{~m} / \mathrm{s}]$ respectively, so that the initial impact velocity remains $12.5[\mathrm{~mm} / \mathrm{s}]$, then simulation of the rigid body displacements predicts that both spheres have been displaced by $17.131[\mathrm{~mm}]$ from their initial position of contact and in the same direction as their initial direction. These rigid body displacements are significant and cannot be neglected as assumed in the classical rigid body theory (Chatterjee, 1997). In fact, the tabulated solutions (see last row of column 4 of Table A2) show that the higher the values of the ratios $V_{i} / V_{0, \text { rel }}$ and $m_{i} / m$, the greater the final rigid body displacement of sphere $i$. 


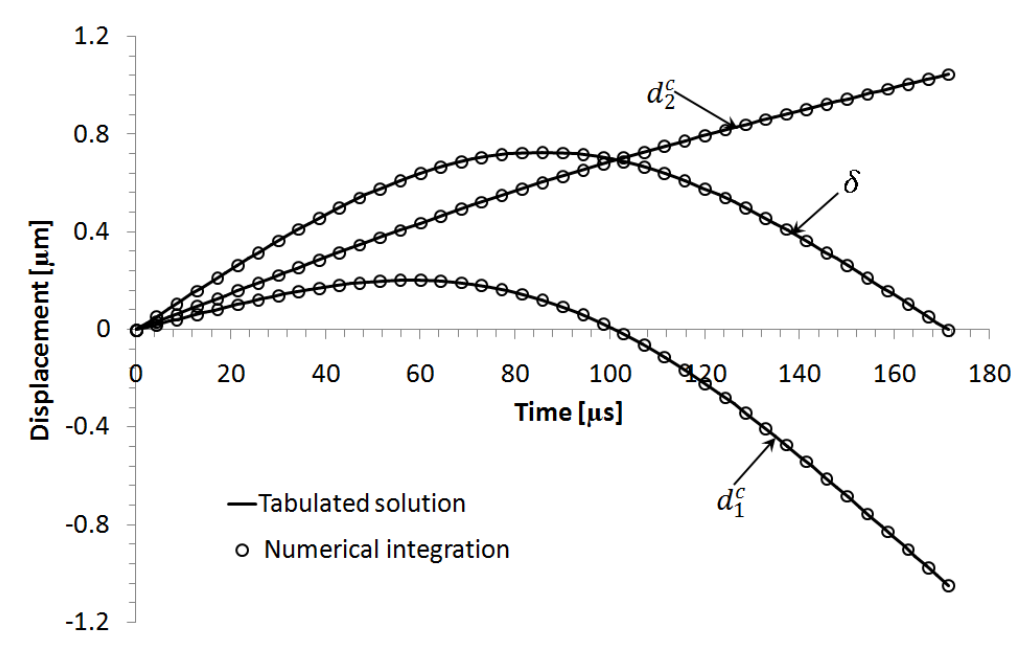

Figure 4. Rigid body displacements - elastic collision

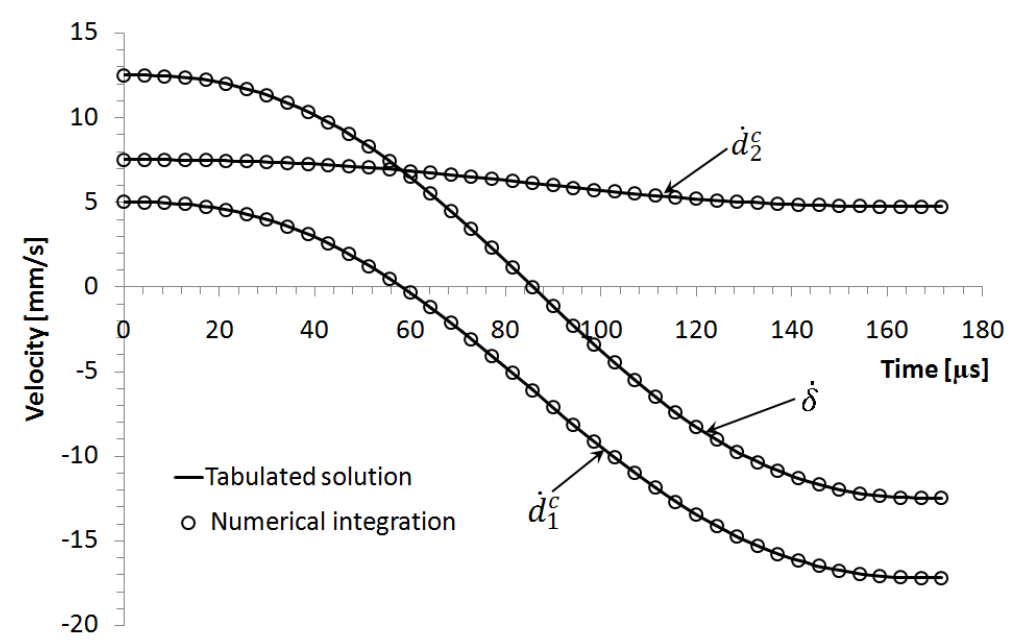

Figure 5. Rigid body velocities - elastic collision

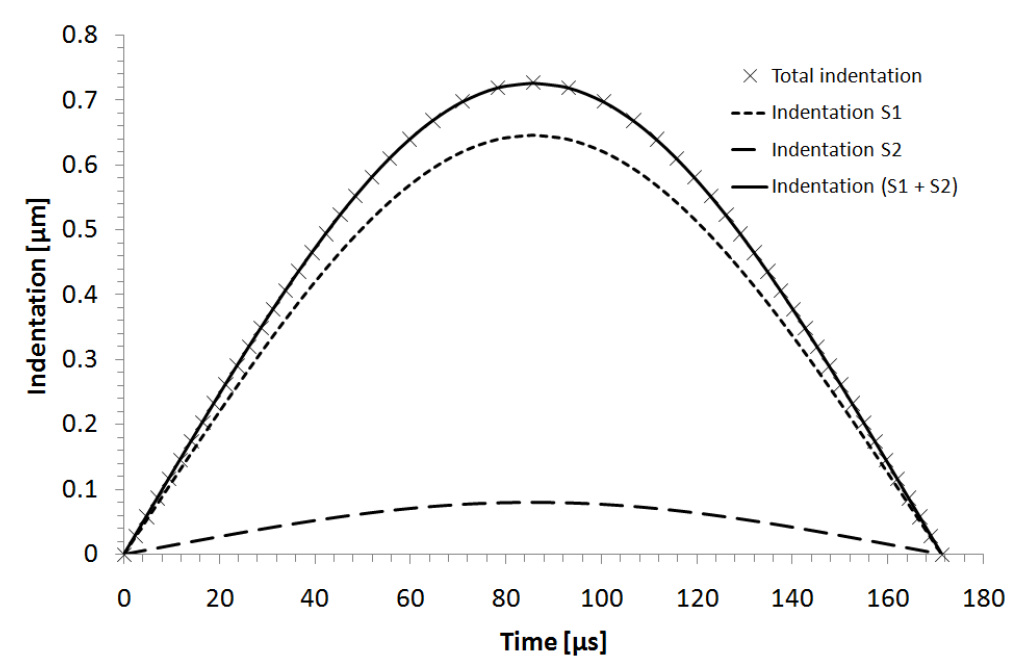

Figure 6. Constituent indentations - elastic collision. S1 and S2 means sphere 1 and sphere 2 


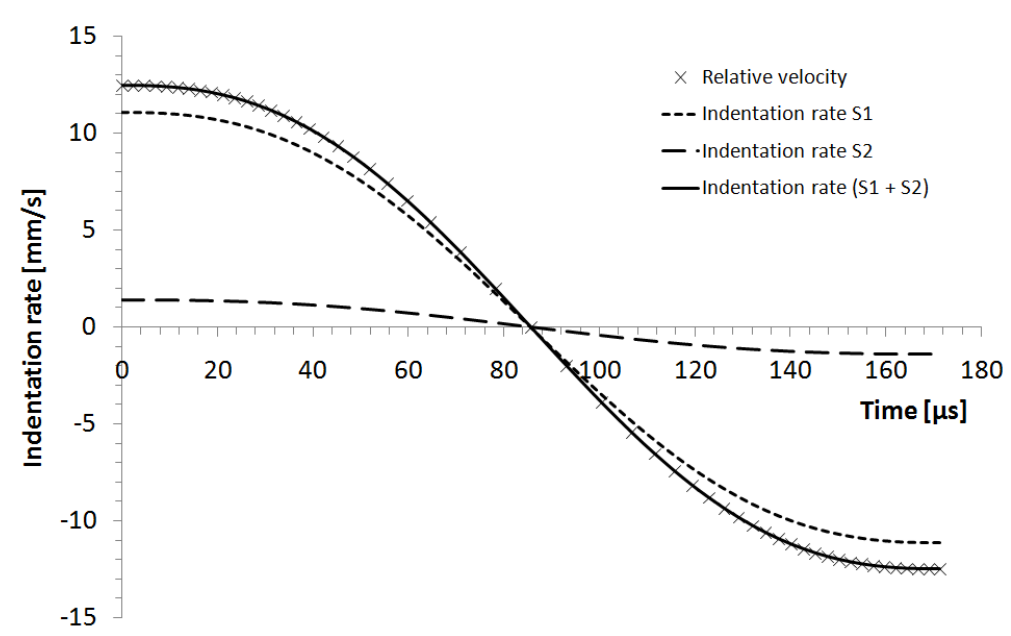

Figure 7. Constituent indentation rates - elastic collision

The two elastic collision problems discussed above have the same initial impact velocity i.e. $12.5[\mathrm{~mm} / \mathrm{s}]$ and therefore, have the same compliance response. Figures 6 and 7 show the compliance responses and confirms that the sum of the constituent compliance responses is equal to the corresponding total compliance response. On the other hand, Figure 8 shows the momentum profiles of the impact process for the first elastic collision problem. It is observed that the total momentum of both spheres is conserved throughout the impact process.

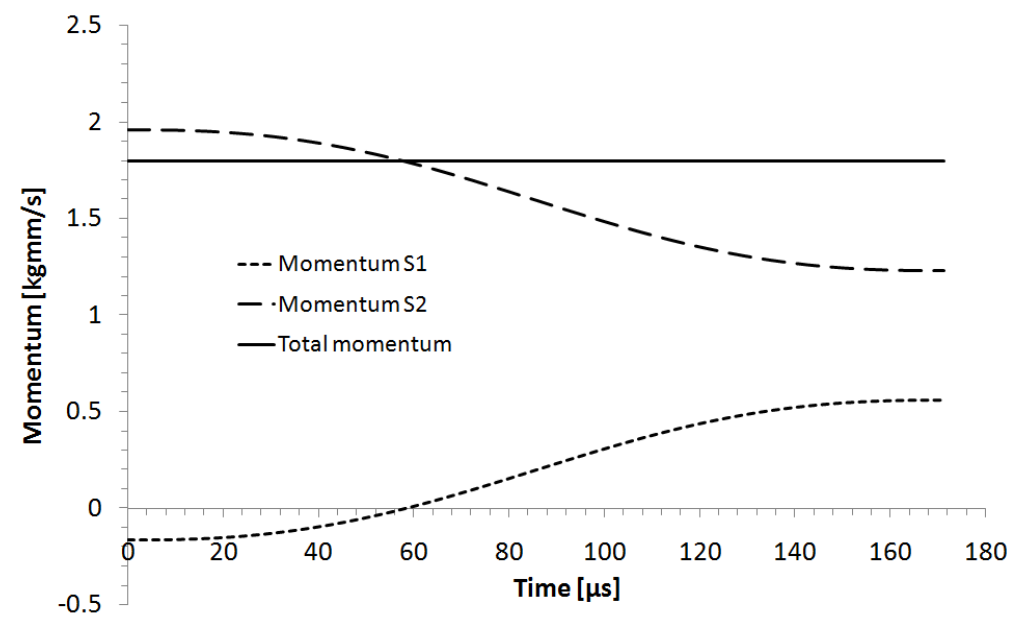

Figure 8. Momentum exchange - elastic collision

\subsection{Example on Normal Elastoplastic Impact of Two Deformable Spheres}

To illustrate elastoplastic impact analysis, the contact model of Du and Wang (2009) is used to estimate the impact force. The force-indentation law for this model is:

$$
F=\left\{\begin{array}{lr}
K_{h} \delta^{3 / 2} & 0 \leq \delta \leq \delta_{y} \\
\pi R p_{0} \delta-\pi^{3} R^{2} p_{0}^{3} /\left(12 E^{2}\right) & \delta_{y} \leq \delta \leq \delta_{m} \\
K_{h}\left(\delta-\delta_{\text {res }}\right)^{3 / 2} & \delta_{\text {res }} \leq \delta \leq \delta_{m}
\end{array}\right\}
$$

where $p_{0}=2.57 \sigma_{y} ; \delta_{y}=\pi^{2} R p_{0}^{2} /\left(4 E^{2}\right)$ and $\delta_{r e s}=\delta_{m}-\left[\frac{\pi R^{1 / 2} p_{0}}{E}\left(\frac{3 \delta_{m}}{4}-\frac{1}{16}\right)\right]^{2 / 3}$.

Equations $16(\mathrm{a}-\mathrm{c})$ are substituted into equation $(1 \mathrm{a}, \mathrm{b})$ and 2 to derive the dynamic models that were solved using the FILM algorithm. The case study considered is similar to the first elastic collision problem discussed in Section 4.3 except that the initial velocities are $0.25[\mathrm{~m} / \mathrm{s}]$ and $0.75[\mathrm{~m} / \mathrm{s}]$ for the spheres 1 and 2 respectively. 
The rigid body displacements and constituent indentation responses are plotted in Figure 9 whereas the rigid body velocities and constituent indentation rates are plotted in Figure 10. The results predict that sphere 1 is displaced $44.61[\mu \mathrm{m}]$ to the right of its initial position of contact (see Figure 9) and has a final velocity of $1.40[\mathrm{~m} / \mathrm{s}]$ opposite to its initial velocity (see Figure 10), while sphere 2 is displaced $49.03[\mu \mathrm{m}]$ to the right of its initial position of contact (see Figure 9) and has a final velocity of $0.54[\mathrm{~m} / \mathrm{s}]$ in the same direction as its initial velocity (see Figure 10). Again, this confirms that the spheres are not stationary during impact.

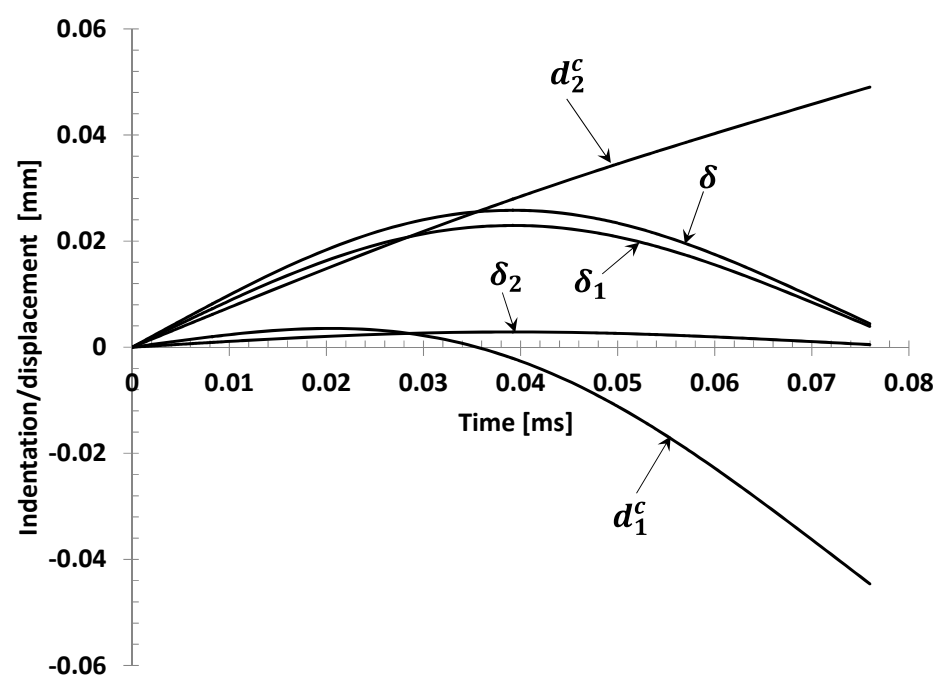

Figure 9. Rigid body displacements and indendations - elastoplastic collision

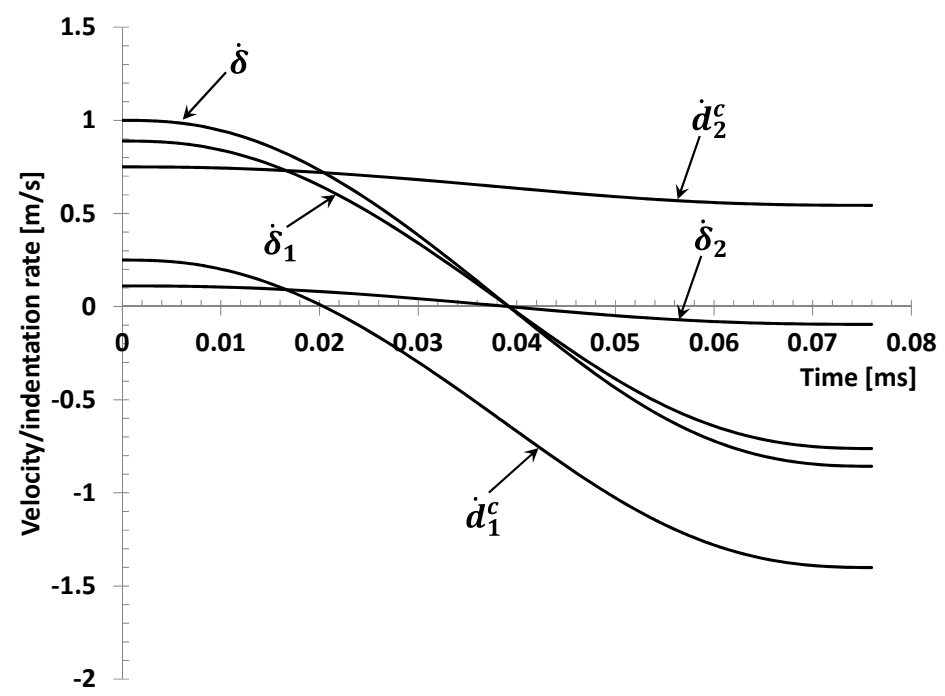

Figure 10. Rigid body velocities and indentation rates - elastoplastic collision

On the other hand, the results of the constituent compliance responses (see Figures 9 and 10) predict that the maximum indentations on spheres 1 and 2 are 22.95[ $\mu \mathrm{m}]$ and 2.87[ $\mu \mathrm{m}]$ respectively, while the corresponding permanent indentations are $3.93[\mu \mathrm{m}]$ and $0.49[\mu \mathrm{m}]$ respectively. These results show that sphere 1 experiences a higher permanent indentation than sphere 2 and the reason is because a higher initial momentum impacts on sphere 1 compared to the initial momentum that impacts on sphere 2 (see Figure 11). The momentum profile in Figure 11 also show that conservation of momentum is not influenced by plastic indentation or the local compliance response. This observation is in agreement with previous collision theory (Stronge, 2000). 


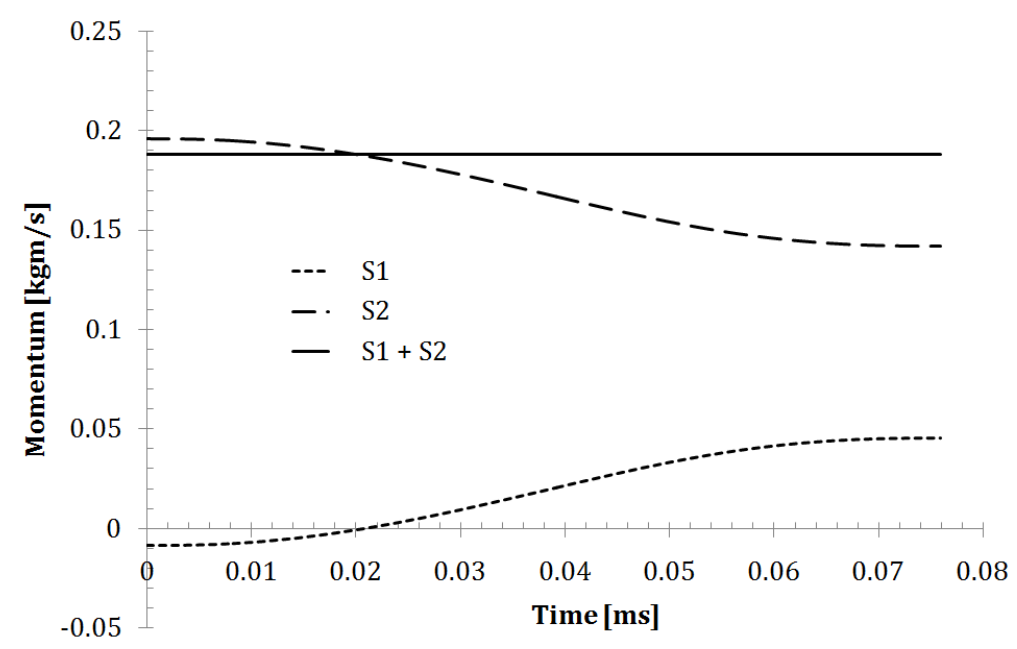

Figure 11. Momentum exchange - elastoplastic collision

\subsection{2-D collision problem}

\subsubsection{Problem Definition}

The 2-D collision problem is one in which two rigid spheres moving in a plane come into collision at an initial angle (see Figures 12a) and separate after collision at a final angle (see Figures $12 \mathrm{~b}$ ). The initial velocities $\left(V_{1}\right.$ and $\left.V_{2}\right)$ and angles $\left(\alpha_{1}\right.$ and $\left.\alpha_{2}\right)$ are known from the problem statement while the final velocities $\left(V_{1 f}\right.$ and $\left.V_{2 f}\right)$ and angles $\left(\theta_{1}\right.$ and $\theta_{2}$ ) are to be determined. Four simultaneous equations are needed to determine the unique solutions to the four unknowns. Based on the widely used classical approach, which is formulated using the rigid body motions before and after collision, the conservation of momentum principle produces two equations (see equations $(17 \mathrm{a}, \mathrm{b})$ )) whereas the conservation of kinetic energy principle produces the third equation (see equation (17c)).

$$
\begin{aligned}
m_{1} V_{1 x}+m_{2} V_{2 x} & =m_{1} V_{1 f x}+m_{2} V_{2 f x} \\
m_{1} V_{1 y}+m_{2} V_{2 y} & =m_{1} V_{1 f y}+m_{2} V_{2 f y} \\
\frac{m_{1}\left(V_{1}\right)^{2}}{2}+\frac{m_{2}\left(V_{2}\right)^{2}}{2} & =\frac{m_{1}\left(V_{1 f}\right)^{2}}{2}+\frac{m_{2}\left(V_{2 f}\right)^{2}}{2}
\end{aligned}
$$
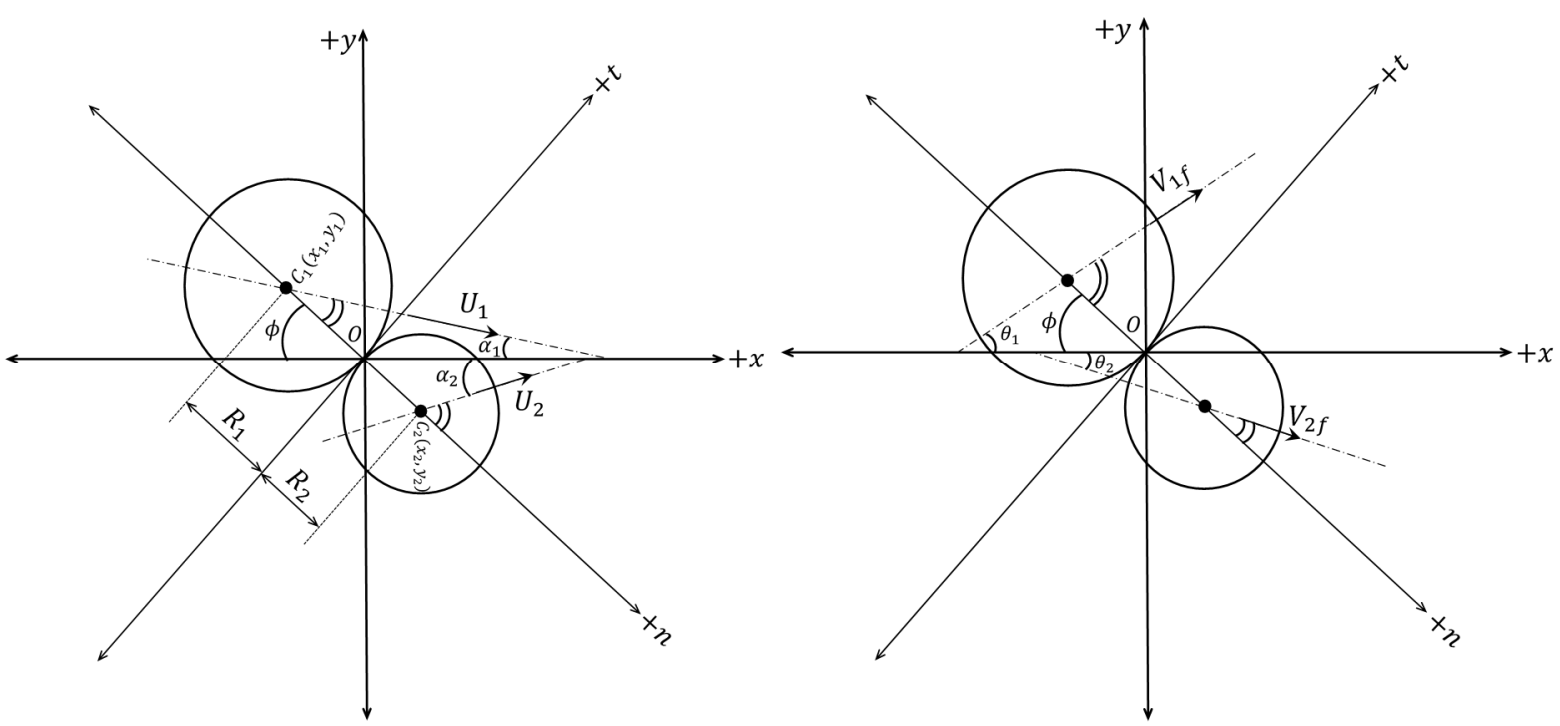

Figure 12. 2-D collision process: (a) onset of collision process (b) end of collision process

where $V_{1 x}=V_{1} \cos \alpha_{1} ; V_{2 x}=V_{2} \cos \alpha_{2} ; V_{1 f x}=V_{1 f} \cos \theta_{1} ; V_{2 f x}=V_{2 f} \cos \theta_{2} ;$ and $V_{1 y}=-V_{1} \sin \alpha_{1} ; V_{2 y}=V_{2} \sin \alpha_{2} ; V_{1 f y}=V_{1} \sin \theta_{1} ; V_{2 f y}=-V_{2} \sin \theta_{2}$. 
Equation (17c) is only applicable to elastic collisions. For elastoplastic collisions, the kinetic energy is not conserved due to dissipation of energy for plastic indentation. The 2-D collision problem discussed here considers only translational motions and assumes that the rotational motions are negligible.

The limitation of the classical approach in solving the 2-D collision problem is immediately obvious because it cannot define the required fourth equation. An alternative approach proposed here is to consider the rigid body motions during collision. Hitherto, the constituent rigid body motions during collision have not been investigated. However, analytical solutions for the constituent rigid body motions during collision have been developed in the present study (see equations $(12 \mathrm{a}, \mathrm{b})$ ) and can be applied to solve the 2-D collision problem as discussed next.

\subsubsection{Solution to 2-D collision problem}

The initial velocity of each sphere can be resolved into normal and tangential components relative to the contact plane. The angles of the initial velocities relative to the contact normal can be used to determine the resolved components. In Figure 12a, these angles are shown using double arcs and are equal to $\phi-\alpha_{1}$ and $\phi+\alpha_{2}$ for $V_{1}$ and $V_{2}$ respectively; where $\phi=\tan ^{-1}\left[\left(y_{1}-y_{2}\right) /\left(x_{1}-x_{2}\right)\right]$ is the inclination of the normal relative to the $\mathrm{x}$-axis. During collision, the position vectors for the centers of mass of the spheres change due rigid body displacement but $\phi$ is unchanged.

From Figure 12a, the normal components of the initial velocities are:

$$
\begin{aligned}
& V_{1 n}=V_{1} \cos \left(\phi-\alpha_{1}\right) \\
& V_{2 n}=V_{2} \cos \left(\phi+\alpha_{2}\right)
\end{aligned}
$$

while the tangential components are:

$$
\begin{aligned}
& V_{1 t}=V_{1} \sin \left(\phi-\alpha_{1}\right) \\
& V_{2 t}=V_{2} \sin \left(\phi+\alpha_{2}\right)
\end{aligned}
$$

At the end of collision, the tangential component of the velocities are unchanged (i.e. $V_{1 f t}=V_{1 t}$ and $V_{2 f t}=V_{2 t}$ ) whereas the normal component of the velocities have changed and their final values are calculated using equation (12a). For purely elastic collision, equation (12a) has been used to derive normalized tabulated solutions from which the final velocities in the normal direction is determined as (see last row of column 5 of Table A2):

$$
V_{i f n}=\left|V_{i n}\right|-2\left(m / m_{i}\right) V_{0 n} \quad \text { for } i=1,2
$$

where $V_{0 n}$ is the initial relative velocity in the normal direction. In equation (20), if $V_{i f n} \geq 0$ then the initial and final directions of sphere $i$ are the same otherwise they are opposite.

With $V_{i f n}$ and $V_{i f t}$ known, the final velocities are calculated as $V_{i f}=\sqrt{\left(V_{i f n}\right)^{2}+\left(V_{i f t}\right)^{2}}$ leaving two unknowns $\theta_{1}$ and $\theta_{2}$, which can be determine by resolving $V_{1 f}$ and $V_{2 f}$ in the normal direction (see Figure 13). Therefore,

$$
\begin{aligned}
& \theta_{1}=\cos ^{-1}\left(V_{1 f n} / V_{1 f}\right)-\phi \\
& \theta_{2}=\phi-\cos ^{-1}\left(V_{2 f n} / V_{2 f}\right)
\end{aligned}
$$

The unique solutions to all four unknowns obtained using the above analysis are accurate if they satisfy equations (17a -c). To illustrate the above solution method, a 2-D elastic collision case study is investigated as shown in the next sub-section.

\subsubsection{Example on 2-D Elastic Collision}

Consider a 2-D elastic collision depicted by Figure 12 for which $\alpha_{1}=30^{\circ}, \alpha_{2}=50^{\circ}, m_{1}=0.05[\mathrm{~kg}], m_{2}=$ $0.15[\mathrm{~kg}], V_{1}=3.0[\mathrm{~m} / \mathrm{s}], V_{2}=2.0[\mathrm{~m} / \mathrm{s}],\left(x_{1}, y_{1}\right)=(-1,2)$ and $\left(x_{2}, y_{2}\right)=(2,-2)$. The calculated final velocities are $2.374[\mathrm{~m} / \mathrm{s}]$ and $2.263[\mathrm{~m} / \mathrm{s}]$ for spheres 1 and 2 respectively, while the calculated final angles relative to the positive $\mathrm{x}$-axis are $97.11^{\circ}$ and $-6.26^{\circ}$ for spheres 1 and 2 respectively. The calculated final velocities and angles satisfy the conservative equations of the classical approach (i.e. equations (17a-c)) perfectly and are therefore accurate.

\section{Conclusions}

In this paper, analytical solutions have been developed to determine the constituent responses of each sphere during normal binary impact of hard deformable spheres. The analytical solutions are applicable to both elastic and elastoplastic collisions and are implemented with the same relative easy irrespective of the complexity of the contact model used to estimate the local compliance. For elastic collisions, tabulated solutions of normalized responses were derived that can be easily used to generate actual impact profiles. Furthermore, the unique solution of all four 
unknowns of the 2-D collision problem is presented based on the analytical solution of the constituent rigid body motions.

The present analysis reveals that the constituent response of each sphere depends on two parameters, namely: (a) ratio of mass of sphere to effective mass of impact system, and (b) ratio of initial velocity of sphere to initial relative velocity. Contrary to widely held believe that the spheres are stationary during collision, the present study shows that the spheres undergo rigid body displacements that cause them to change their position during collision. It was also observed that the total momentum of the impact system is conserved throughout the impact process and is independent of the local compliance.

This study focuses on the collision period and improves understanding of the phenomenon of rigid body collision. The present study is important for the following reasons:

1) It can be used to determine the indentation response of each body in a binary collision and to predict the permanent indentation realised on each body, if any. Such information can assist to design experiments in which it is desired that one of the colliding bodies does not undergo elastoplastic indentation (Kharaz and Gorham, 2000).

2) It provides information for accurate motion tracking of freely colliding particles as found in atomic collisions and granular flow processes. The analysis presented in this paper shows that the colliding spheres undergo individual rigid body motions and the extent of these motions can be estimated.

3) The energy utilisation mechanism of the collision process can now be understood better because the constituent local compliance and rigid body motions can be determined based on present study. This means that the energy absorbed by each sphere for its local indentation and the energy utilised by each sphere for its rigid body motion can be analysed.

4) The solution of the rigid body motions makes it possible to obtain unique solutions for 2-D elastic and elastoplastic collision problems.

\section{Acknowledgments}

No grant or funding was received for this research.

\section{References}

Andrews, J. P. (1930a). Experiments on impact. Proceedings of Physical Society, 43, 8-17. https://oi.org/10.1088/ 0959-5309/43/1/303.

Andrews, J. P. (1930b). Theory of collision of spheres of soft metals. Philosophical Magazine: Series 7, 595-610.

Big-Alabo, A., Harrison P., \& Cartmell, M. P. (2015). Algorithm for the solution of elastoplastic half-space impact. Force-Indentation Linearisation Method, Journal of Mechanical Engineering Sciences, 229(5), 850-858, https://doi.org/10.1177/0954406214541431

Big-Alabo, A., Cartmell, M. P., \& Harrison, P. (2017). On the solution of asymptotic impact problems with significant localised indentation, Journal of Mechanical Engineering Sciences, 231(5), 807-822, https://doi.org/10.1177/ 0954406216628556.

Campbell, C. S. (2006). Granular material flows - An overview. Powder Technology, 162, 208-229. https://doi.org/10. 1016/j.powtec.2005.12.008.

Chatterjee, A. (1997). Rigid body collision. Some general considerations, new collision laws, and some experimental data. PhD Thesis, Cornell University, United States, p21.

Coaplen, J., Stronge, W. J., \& Ravani, B. (2004). Work equivalent composite coefficient of restitution, International Journal of Impact Engineering, 30, 581-591, https://doi.org/10.1016/j.ijimpeng.2003.10.038

Du, Y., \& Wang, S. (2009). Energy dissipation in normal elastoplastic impact between two spheres. ASME Transactions: Journal of Applied Mechanics, 76(6), 061010, https://doi.org/10.1115/1.3130801

Goldsmith, W. (2001). Impact: The theory and physical behaviour of colliding solids. New York: Dover Publications.

Johnson, K. L. (1985). Contact mechanics. Cambridge: Cambridge University Press. https://doi.org/10.1017/ CBO9781139171731.

Kharaz, A. H., \& Gorham, D. A. (2000). A study of coefficient of restitution in elastic-plastic impact. Philosophical Magazine Letters, 80(8), 549-559. https://doi.org/10.1080/09500830050110486 
Machado, M., Moreira, P., Flores, P., \& Lankarani, H. M. (2012). Compliant contact force models in multibody dynamics: Evolution of the Hertz contact theory, Mechanisms and Machine Theory, 53, 99-121, https://doi.org/10.1016/j.mechmachtheory.2012.02.010.

Minamoto, H., \& Kawamura, S. (2009). Effects of material strain rate sensitivity in low speed impact between two identical spheres. International Journal of Impact Engineering, 36, 680-686, https://doi.org/10.1016/j.ijimpeng. 2008.10.001

Minamoto, H., \& Kawamura, S. (2011). Moderately high speed impact of two identical spheres, International Journal of Impact Engineering, 38, 123-129. https://doi.org/10.1016/j.ijimpeng.2010.09.005

Stronge, W. J. (2000). Impact mechanics. Cambridge: Cambridge University Press, https://doi.org/10.1017/ CBO9780511626432.

\section{Appendix}

\section{Tabulated solutions for elastic impact response of two hard deformable spheres}

Table A1. Normalized solutions for total compliance response and constituent rigid body motions - loading stage

\begin{tabular}{|c|c|c|c|c|c|}
\hline$t\left(\frac{V_{0, r e l}}{\delta_{m}}\right)$ & $\frac{\delta}{\delta_{m}}$ & $\frac{\dot{\delta}}{V_{0, \text { rel }}}$ & $\frac{d_{i}^{c}}{\delta_{m}}(E q n .14 b)$ & $\frac{\dot{d}_{i}^{c}}{V_{0, r e l}}(E q n .14 a)$ & $\frac{F}{F_{m}}$ \\
\hline 0.0000 & 0.0000 & 1.0000 & 0.00000 & $\bar{V}_{i}$ & 0.0000 \\
\hline 0.0400 & 0.0400 & 0.9998 & $0.0400027 \bar{V}_{i}-2.66715 \times 10^{-6} \bar{m}_{i}^{-1}$ & $\bar{V}_{i}-0.000160013 \bar{m}_{i}^{-1}$ & 0.0080 \\
\hline 0.0800 & 0.0800 & 0.9991 & $0.080022 \bar{V}_{i}-0.0000219599 \bar{m}_{i}^{-1}$ & $\bar{V}_{i}-0.000905507 \bar{m}_{i}^{-1}$ & 0.0226 \\
\hline 0.1201 & 0.1200 & 0.9975 & $0.120087 \bar{V}_{i}-0.0000872777 \bar{m}_{i}^{-1}$ & $\bar{V}_{i}-0.00249727 \bar{m}_{i}^{-1}$ & 0.0416 \\
\hline 0.1602 & 0.1600 & 0.9949 & $0.160237 \bar{V}_{i}-0.000236961 \bar{m}_{i}^{-1}$ & $\bar{V}_{i}-0.00513317 \bar{m}_{i}^{-1}$ & 0.0640 \\
\hline 0.2005 & 0.2000 & 0.9910 & $0.200517 \bar{V}_{i}-0.000517235 \bar{m}_{i}^{-1}$ & $\bar{V}_{i}-0.00898463 \bar{m}_{i}^{-1}$ & 0.0894 \\
\hline 0.2410 & 0.2400 & 0.9858 & $0.240982 \bar{V}_{i}-0.000981938 \bar{m}_{i}^{-1}$ & $\bar{V}_{i}-0.01421 m_{i}^{-1}$ & 0.1176 \\
\hline 0.2817 & 0.2800 & 0.9790 & $0.281693 \bar{V}_{i}-0.00169281 \bar{m}_{i}^{-1}$ & $\bar{V}_{i}-0.0209624 \bar{m}_{i}^{-1}$ & 0.1482 \\
\hline 0.3227 & 0.3200 & 0.9706 & $0.32272 \bar{V}_{i}-0.00272026 \bar{m}_{i}^{-1}$ & $\bar{V}_{i}-0.0293951 \bar{m}_{i}^{-1}$ & 0.1810 \\
\hline 0.3641 & 0.3600 & 0.9603 & $0.364145 \bar{V}_{i}-0.00414462 \bar{m}_{i}^{-1}$ & $\bar{V}_{i}-0.0396667 \bar{m}_{i}^{-1}$ & 0.2160 \\
\hline 0.4061 & 0.4000 & 0.9481 & $0.406058 \bar{V}_{i}-0.00605792 \bar{m}_{i}^{-1}$ & $\bar{V}_{i}-0.0519456 \bar{m}_{i}^{-1}$ & 0.2530 \\
\hline 0.4486 & 0.4400 & 0.9336 & $0.448566 \bar{V}_{i}-0.00856645 \bar{m}_{i}^{-1}$ & $\bar{V}_{i}-0.0664154 \bar{m}_{i}^{-1}$ & 0.2919 \\
\hline 0.4918 & 0.4800 & 0.9167 & $0.491794 \bar{V}_{i}-0.0117942 \bar{m}_{i}^{-1}$ & $\bar{V}_{i}-0.0832807 \bar{m}_{i}^{-1}$ & 0.3326 \\
\hline 0.5359 & 0.5200 & 0.8972 & $0.535888 \bar{V}_{i}-0.0158877 \bar{m}_{i}^{-1}$ & $\bar{V}_{i}-0.102776 \bar{m}_{i}^{-1}$ & 0.3750 \\
\hline 0.5810 & 0.5600 & 0.8748 & $0.581023 \bar{V}_{i}-0.0210228 \bar{m}_{i}^{-1}$ & $\bar{V}_{i}-0.125172 \bar{m}_{i}^{-1}$ & 0.4191 \\
\hline 0.6274 & 0.6000 & 0.8492 & $0.627414 \bar{V}_{i}-0.027414 \bar{m}_{i}^{-1}$ & $\bar{V}_{i}-0.150797 \bar{m}_{i}^{-1}$ & 0.4648 \\
\hline 0.6753 & 0.6400 & 0.8200 & $0.675329 \bar{V}_{i}-0.0353292 \bar{m}_{i}^{-1}$ & $\bar{V}_{i}-0.180049 \bar{m}_{i}^{-1}$ & 0.5120 \\
\hline 0.7251 & 0.6800 & 0.7866 & $0.725111 \bar{V}_{i}-0.0451107 \bar{m}_{i}^{-1}$ & $\bar{V}_{i}-0.213428 \bar{m}_{i}^{-1}$ & 0.5607 \\
\hline 0.7772 & 0.7200 & 0.7484 & $0.77721 \bar{V}_{i}-0.0572103 \bar{m}_{i}^{-1}$ & $\bar{V}_{i}-0.251586 \bar{m}_{i}^{-1}$ & 0.6109 \\
\hline 0.8322 & 0.7600 & 0.7046 & $0.832246 \bar{V}_{i}-0.0722462 \bar{m}_{i}^{-1}$ & $\bar{V}_{i}-0.295401 \bar{m}_{i}^{-1}$ & 0.6626 \\
\hline 0.8911 & 0.8000 & 0.6539 & $0.891108 \bar{V}_{i}-0.0911075 \bar{m}_{i}^{-1}$ & $\bar{V}_{i}-0.346114 \bar{m}_{i}^{-1}$ & 0.7155 \\
\hline 0.9552 & 0.8400 & 0.5944 & $0.955159 \bar{V}_{i}-0.11516 \bar{m}_{i}^{-1}$ & $\bar{V}_{i}-0.405604 \bar{m}_{i}^{-1}$ & 0.7699 \\
\hline 1.0267 & 0.8800 & 0.5230 & $1.0267 \bar{V}_{i}-0.1467 \bar{m}_{i}^{-1}$ & $\bar{V}_{i}-0.476981 \bar{m}_{i}^{-1}$ & 0.8255 \\
\hline 1.1102 & 0.9200 & 0.4338 & $1.11023 \bar{V}_{i}-0.19023 \bar{m}_{i}^{-1}$ & $\bar{V}_{i}-0.566224 \bar{m}_{i}^{-1}$ & 0.8824 \\
\hline 1.2174 & 0.9600 & 0.3115 & $1.21739 \bar{V}_{i}-0.25739 \bar{m}_{i}^{-1}$ & $\bar{V}_{i}-0.68852 \bar{m}_{i}^{-1}$ & 0.9406 \\
\hline 1.3445 & 0.9899 & 0.1581 & $1.34451 \bar{V}_{i}-0.35461 \bar{m}_{i}^{-1}$ & $\bar{V}_{i}-0.84187 \bar{m}_{i}^{-1}$ & 0.9849 \\
\hline 1.4716 & 1.0000 & 0.0000 & $1.47163 \bar{V}_{i}-0.47163 \bar{m}_{i}^{-1}$ & $\bar{V}_{i}-1.00000 \bar{m}_{i}^{-1}$ & 1.0000 \\
\hline
\end{tabular}


Table A2. Normalized solutions for total compliance response and constituent rigid body motions- restitution stage

\begin{tabular}{|c|c|c|c|c|c|}
\hline$t\left(\frac{V_{0, r e l}}{\delta_{m}}\right)$ & $\frac{\delta}{\delta_{m}}$ & $\frac{\dot{\delta}}{V_{0, \text { rel }}}$ & $\frac{d_{i}^{c}}{\delta_{m}}(E q n .14 b)$ & $\frac{\dot{d}_{i}^{c}}{V_{0, \text { rel }}}($ Eqn.14a) & $\frac{F}{F_{m}}$ \\
\hline 1.4716 & 1.0000 & 0.0000 & $1.47163 \bar{V}_{i}-0.47163 \bar{m}_{i}^{-1}$ & $\bar{V}_{i}-1.00000 \bar{m}_{i}^{-1}$ & 1.0000 \\
\hline 1.5988 & 0.9899 & -0.1581 & $1.59875 \bar{V}_{i}-0.60885 \bar{m}_{i}^{-1}$ & $\bar{V}_{i}-1.15814 \bar{m}_{i}^{-1}$ & 0.9849 \\
\hline 1.7259 & 0.9600 & -0.3115 & $1.72587 \bar{V}_{i}-0.76587 \bar{m}_{i}^{-1}$ & $\bar{V}_{i}-1.31148 \bar{m}_{i}^{-1}$ & 0.9406 \\
\hline 1.8330 & 0.9200 & -0.4338 & $1.83302 \bar{V}_{i}-0.91302 \bar{m}_{i}^{-1}$ & $\bar{V}_{i}-1.43378 \bar{m}_{i}^{-1}$ & 0.8824 \\
\hline 1.9165 & 0.8800 & -0.5230 & $1.91653 \bar{V}_{i}-1.03653 \bar{m}_{i}^{-1}$ & $\bar{V}_{i}-1.52302 \bar{m}_{i}^{-1}$ & 0.8255 \\
\hline 1.9881 & 0.8400 & -0.5944 & $1.98807 \bar{V}_{i}-1.14807 \bar{m}_{i}^{-1}$ & $\bar{V}_{i}-1.59440 \bar{m}_{i}^{-1}$ & 0.7699 \\
\hline 2.0521 & 0.8000 & -0.6539 & $2.05211 \bar{V}_{i}-1.25211 \bar{m}_{i}^{-1}$ & $\bar{V}_{i}-1.65389 \bar{m}_{i}^{-1}$ & 0.7155 \\
\hline 2.1110 & 0.7600 & -0.7046 & $2.11097 \bar{V}_{i}-1.35097 \bar{m}_{i}^{-1}$ & $\bar{V}_{i}-1.70460 \bar{m}_{i}^{-1}$ & 0.6626 \\
\hline 2.1660 & 0.7200 & -0.7484 & $2.16600 \bar{V}_{i}-1.44600 \bar{m}_{i}^{-1}$ & $\bar{V}_{i}-1.74841 \bar{m}_{i}^{-1}$ & 0.6109 \\
\hline 2.2181 & 0.6800 & -0.7866 & $2.21810 \bar{V}_{i}-1.53810 \bar{m}_{i}^{-1}$ & $\bar{V}_{i}-1.78657 \bar{m}_{i}^{-1}$ & 0.5607 \\
\hline 2.2679 & 0.6400 & -0.8200 & $2.26787 \bar{V}_{i}-1.62787 \bar{m}_{i}^{-1}$ & $\bar{V}_{i}-1.81995 \bar{m}_{i}^{-1}$ & 0.5120 \\
\hline 2.3158 & 0.6000 & -0.8492 & $2.31579 \bar{V}_{i}-1.71579 \bar{m}_{i}^{-1}$ & $\bar{V}_{i}-1.84920 \bar{m}_{i}^{-1}$ & 0.4648 \\
\hline 2.3622 & 0.5600 & -0.8748 & $2.36217 \bar{V}_{i}-1.80217 \bar{m}_{i}^{-1}$ & $\bar{V}_{i}-1.87483 \bar{m}_{i}^{-1}$ & 0.4191 \\
\hline 2.4073 & 0.5200 & -0.8972 & $2.40731 \bar{V}_{i}-1.88731 \bar{m}_{i}^{-1}$ & $\bar{V}_{i}-1.89722 \bar{m}_{i}^{-1}$ & 0.3750 \\
\hline 2.4514 & 0.4800 & -0.9167 & $2.45140 \bar{V}_{i}-1.97140 \bar{m}_{i}^{-1}$ & $\bar{V}_{i}-1.91672 \bar{m}_{i}^{-1}$ & 0.3326 \\
\hline 2.4946 & 0.4400 & -0.9336 & $2.49462 \bar{V}_{i}-2.05462 \bar{m}_{i}^{-1}$ & $\bar{V}_{i}-1.93358 \bar{m}_{i}^{-1}$ & 0.2919 \\
\hline 2.5371 & 0.4000 & -0.9481 & $2.53713 \bar{V}_{i}-2.13713 \bar{m}_{i}^{-1}$ & $\bar{V}_{i}-1.94805 \bar{m}_{i}^{-1}$ & 0.2530 \\
\hline 2.5790 & 0.3600 & -0.9603 & $2.57904 \bar{V}_{i}-2.21904 \bar{m}_{i}^{-1}$ & $\bar{V}_{i}-1.96033 \bar{m}_{i}^{-1}$ & 0.2160 \\
\hline 2.6205 & 0.3200 & -0.9706 & $2.62046 \bar{V}_{i}-2.30046 \bar{m}_{i}^{-1}$ & $\bar{V}_{i}-1.97060 \bar{m}_{i}^{-1}$ & 0.1810 \\
\hline 2.6615 & 0.2800 & -0.9790 & $2.66149 \bar{V}_{i}-2.38149 \bar{m}_{i}^{-1}$ & $\bar{V}_{i}-1.97904 \bar{m}_{i}^{-1}$ & 0.1482 \\
\hline 2.7022 & 0.2400 & -0.9858 & $2.70220 \bar{V}_{i}-2.46220 \bar{m}_{i}^{-1}$ & $\bar{V}_{i}-1.98579 \bar{m}_{i}^{-1}$ & 0.1176 \\
\hline 2.7427 & 0.2000 & -0.9910 & $2.74266 \bar{V}_{i}-2.54266 \bar{m}_{i}^{-1}$ & $\bar{V}_{i}-1.99102 \bar{m}_{i}^{-1}$ & 0.0894 \\
\hline 2.7829 & 0.1600 & -0.9949 & $2.78294 \bar{V}_{i}-2.62294 \bar{m}_{i}^{-1}$ & $\bar{V}_{i}-1.99487 \bar{m}_{i}^{-1}$ & 0.0640 \\
\hline 2.8231 & 0.1200 & -0.9975 & $2.82308 \bar{V}_{i}-2.70308 \bar{m}_{i}^{-1}$ & $\bar{V}_{i}-1.99750 \bar{m}_{i}^{-1}$ & 0.0416 \\
\hline 2.8632 & 0.0800 & -0.9991 & $2.86315 \bar{V}_{i}-2.78315 \bar{m}_{i}^{-1}$ & $\bar{V}_{i}-1.99909 \bar{m}_{i}^{-1}$ & 0.0226 \\
\hline 2.9032 & 0.0400 & -0.9998 & $2.90317 \bar{V}_{i}-2.86317 \bar{m}_{i}^{-1}$ & $\bar{V}_{i}-1.99984 \bar{m}_{i}^{-1}$ & 0.0080 \\
\hline 2.9432 & 0.0000 & -1.0000 & $2.94317 \bar{V}_{i}-2.94317 \bar{m}_{i}^{-1}$ & $\bar{V}_{i}-2.00000 \bar{m}_{i}^{-1}$ & 0.0000 \\
\hline
\end{tabular}

Note: $\bar{V}_{i}=V_{i} / V_{0, r e l}$ and $\bar{m}_{i}=m_{i} / m$.

\section{Copyrights}

Copyright for this article is retained by the author(s), with first publication rights granted to the journal.

This is an open-access article distributed under the terms and conditions of the Creative Commons Attribution license (http://creativecommons.org/licenses/by/4.0/). 